\title{
Effects of Fluorine Atoms Amount and Fluorinated Acrylic Chain Length Chemically Attached to Hydroxyl Groups on the Hydrophobic Properties of Cotton Fabrics
}

\author{
Liu $\mathbf{X}^{1}$, Yang $\mathbf{G}^{1}$ and Lipik $\mathbf{V T}^{2 *}$ \\ ${ }^{1}$ Institute for Sport Research, Nanyang Technological University, Singapore \\ ${ }^{2}$ School of Material Science and Engineering, Nanyang Technological University, Singapore
}

\begin{abstract}
A two-step chemical treatment of cotton fabric was performed with the attachment of fluorine moieties on hydroxyl group sites. The hydroxyl groups of cotton were initially acrylated by 2 -isocyanaethyl methacrylate. Acrylic monomers, containing four to twelve fluorine atoms, had been used to build polymeric chain directly on the surface of cotton by means of radical polymerization. Fabrics became $10-20 \%$ stiffer and microscopy showed a clear change of the cotton surface after the treatment. Coated samples of cotton had shown hydrophobic property with a highest contact angle of 128 degrees. It was found that the increase of molecular weight of fluorinated polyacrylate on the cotton surface lowered contact angle value. The best results in hydrophobicity had been obtained at the molar stoichiometric ratio between the number of hydroxyl groups of cotton and the amount of fluorinated monomer added. This developed method allowed for the direct radical polymerization on cotton fabric, providing good hydrophobic properties with the formation of fluorinated polyacrylate of different molecular weights.
\end{abstract}

Keywords: Hydrophobicity; Fluorinated coating; Radical polymerization; Fluorinated acrylates; Contact angle

\section{Introduction}

Cotton is a popular natural fiber for making comfortable apparel. However, cotton absorbs water due to its abundance of hydroxyl ($\mathrm{OH})$ groups, thus reducing its mechanical and thermoinsulation properties. Various methods have been used to obtain water repellency on cotton surfaces, such as coating with water-resistant materials like nanoparticles [1] paraffin wax [2] silicon resin [3] or fluorocarbon [4]. Coating via the chemical transformation of $-\mathrm{OH}$ group is the widely known method for enhancing water resistance of cotton fibers and fabrics. Chemical transformation of $-\mathrm{OH}$ groups has some disadvantages such as long reaction time, involvement of non-ecologically friendly chemicals or solvents, necessity of special reactors as compared to methods using simple wetting in emulsions or coating with polymers. However, chemical transformation of $-\mathrm{OH}$ groups of cotton provides a better control of the process and higher washing durability as compared to physical coating due to formations of chemical bonds between cotton surfaces and water-repellent agents. These water-repellent agents could be in the forms of single molecules, oligomers or polymers. Among known chemical methods, fluorine is the best element to be used to lower the surface free energy and make fabric hydrophobic $[5,6]$. Fluorine has a small radius and a high electronegativity, thus the covalent bond between fluorine and carbon is extremely stable. When fluorine is replaced by other elements such as $\mathrm{H}$ and $\mathrm{C}$, in the order $-\mathrm{CF}_{3}<\mathrm{CF}_{2} \mathrm{H}<\mathrm{CF}_{2}<\mathrm{CH}_{3}<\mathrm{CH}_{2}$, the surface free energy is increased. Therefore, at the present, the impregnation of textiles with fluorine containing polymer dispersions or solutions is the most applied technique [7]. The increase in the number of fluorine-carbon bonds in the functional group attached to cotton will result in lower surface energy and higher hydrophobicity [8]. However, it has limitations such as poor adhesion between the substrate and the fluorine-based hydrophobic coating due to the weak van der Waals force [9]. Hence, chemical attachment of fluorinated water repellent agent to a cotton surface is a prominent direction that allows for the creation of a stable hydrophobic surface. And fluorinated acrylates are the most common and convenient substances for hydrophobic coating [10]. Currently, chemical attachment of fluorine-containing agents to the cotton surface is being developed by three different approaches. The first direction encompasses synthesis of various small molecules and polymers, which can react with $\mathrm{OH}$ groups on the cotton surface. For example, Schondelmaier [11] had synthesized fluoroalkylsilane molecules with hydrophobic and hydrophilic parts, where the hydrophilic parts of the molecules react with $-\mathrm{OH}$ groups. An interesting acrylate-based fluorinated agent with six fluorine atoms monomer had also been synthesized by Yang [12]. The chemistry was realized in three steps and the final product has shown good water-repellent properties when applied on cotton fabrics. A clear benefit for industrial application is that this treatment of cotton can be realized within a short time. A good water-repellent property was obtained at the usage of diblock copolymer in which one part provides water-repellent property whereas another part bearing alkoxysilane or epoxyde group can be chemically attached to the $\mathrm{OH}$ group on the cotton surface [13]. The second approach consists of the development of one-step methods of the fluorinated coating of cotton fabric. For example, a one-step process of attachment of a fluorinated monomer to the surface of cotton had been shown by Cai [14] who used irradiation-induced graft polymerization for direct deposition of nonafluorohexyl-1-acrylate on the cotton surface. In another example, direct fluorination of cotton had been done using elemental fluorine [15]. Another direction consists in the modification of $-\mathrm{OH}$ groups on a cotton surface into other functional groups, which are to be used for attachment of different water repellent agent, can be done. For example, $\mathrm{Yu}$ [16] successfully oxidized the primary -OH groups of cotton by laccase/TEMPO treatment, obtaining aldehyde instead of $\mathrm{OH}$ group, which were then used for the reaction with octadecylamine.

*Corresponding author: Vitali L, School of Material Science and Engineering, Nanyang Technological University, Singapore, Tel: +6583792515; E-mail: vitali@ntu.edu.sg

Received December 21, 2016; Accepted January 02, 2017; Published January 06, 2017

Citation: Liu X, Yang G, Lipik VT (2017) Effects of Fluorine Atoms Amount and Fluorinated Acrylic Chain Length Chemically Attached to Hydroxyl Groups on the Hydrophobic Properties of Cotton Fabrics. Mod Chem Appl 5: 204. doi: 10.4172/2329-6798.1000204

Copyright: @ 2017 Liu X, et al. This is an open-access article distributed under the terms of the Creative Commons Attribution License, which permits unrestricted use, distribution, and reproduction in any medium, provided the original author and source are credited. 
In the first deep work, devoted to fluorination of cotton [17], authors studied hydrophobic property of cotton coated by fluorocontaining chemicals with chain length up to $\mathrm{C} 19$, which were fixed on the cotton surface by different resins. In our work, following the above-mentioned third approach, we, targeting to make wider its possibility, modified the cotton surface, transforming - $\mathrm{OH}$ groups into acrylic functional groups. This allows for the further polymerization of fluorinated acrylic monomers and the attachment of macromolecules directly onto the surface of a cotton fabric with variation of chain length of fluorinated polymer.

\section{Materials and Methods}

\section{Chemicals}

The chemicals, 2-isocyanatoethyl methacrylate, 2,2,3,3-Tetrafluorofluoropropyl methacrylate (TFPA), 2,2,3,4,4,4-Hexafluorofluorobutyl acrylate (HFBA), 2,2,3,4,4,5,5-Octafluoropentyl acrylate (OFPA) and 2,2,3,3,4,4,5,5,5,6,6,7,7-Dodecafluoroheptyl acrylate (DFHA), dibutyltin dilaurate, azobisisobutyronitrile (AIBN), dichloromethane and dimethylformamide were purchased from Sigma-Aldrich. All chemicals were used as received.

\section{Modification of cotton fabric}

Plain woven cotton fabric samples, with an area of about $25 \mathrm{~cm}^{2}$ each, were washed three times with distilled water and dried at $120^{\circ} \mathrm{C}$ for 2 hours before use. Four different fluorinated acrylic monomers of different chain lengths were used to investigate the effect that chain lengths of fluorine-containing acrylic attached to a cotton surface has on hydrophobicity. The monomers were attached via a two-step chemical reaction. The first step is acrylation, where $-\mathrm{OH}$ groups on cotton are transformed into an acrylic group. The second step is radical polymerization, where the fluorinated monomers are polymerized and attached, forming macromolecules on the cotton surface.

Acrylation of cotton: The modification of -OH groups of the cotton into acrylic had been done with reference to the published procedure (Scheme 1). For the synthesis of the intermediate product, cotton fabric strips measuring $2 \mathrm{~cm}$ by $5 \mathrm{~cm}$ were immersed in dichloromethane solvent in a two-neck flask, followed by addition of 2-isocyanatoethyl methacrylate (acrylating agent) and dibutyltin dilaurate catalyst [18]. The molar ratio of $-\mathrm{OH}$ groups of cotton to acrylating agent was 1:10 and the amount of catalyst added was $1 \%$ of the molar amount of the acrylating agent. The two-neck flask was kept under nitrogen gas for 5 days under room temperature after which the cotton fabric was rinsed with ethanol and water multiple times. The cotton samples were then dried in the oven at $70^{\circ} \mathrm{C}$ for 24 hours.

Radical polymerization and the attachment of fluorinated monomers onto cotton surface: Fluorinated monomers with different amounts of fluorine (2,2,3,3-Tetrafluorofluoropropyl methacrylate, 2,2,3,4,4,4-Hexafluorofluorobutyl acrylate, 2,2,3,4,4,5,5-Octafluoropentyl acrylate and 2,2,3,3,4,4,5,5,5,6,6,7,7-Dodecafluoroheptyl acrylate) were attached to an acrylic group on the fabric via radical polymerization (Scheme 2). Polymerization had been done in dimethylformamide for 24 hours at $70^{\circ} \mathrm{C}$. Three different ratios $(1: 1,1: 5,1: 10)$ of acrylic groups on the cotton surface to fluorinated acrylate monomers had been used in the experiment, targeting polymers with different molecular weights attached on the cotton surface. The quantity of fluorinated monomers was calculated under the assumption that all the $-\mathrm{OH}$ groups of cotton had been acrylated during the treatment with 2-isocyanatoethyl methacrylate. The amount of catalyst - AIBN - remained constant for all experiments at a ratio of 1:50 to the molar amount of monomer taken for the polymerization. After polymerization, the

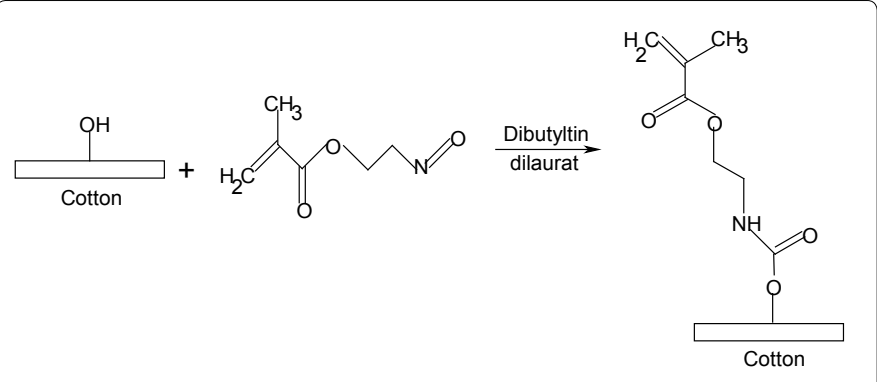

Scheme 1: Acrylation of cotton.

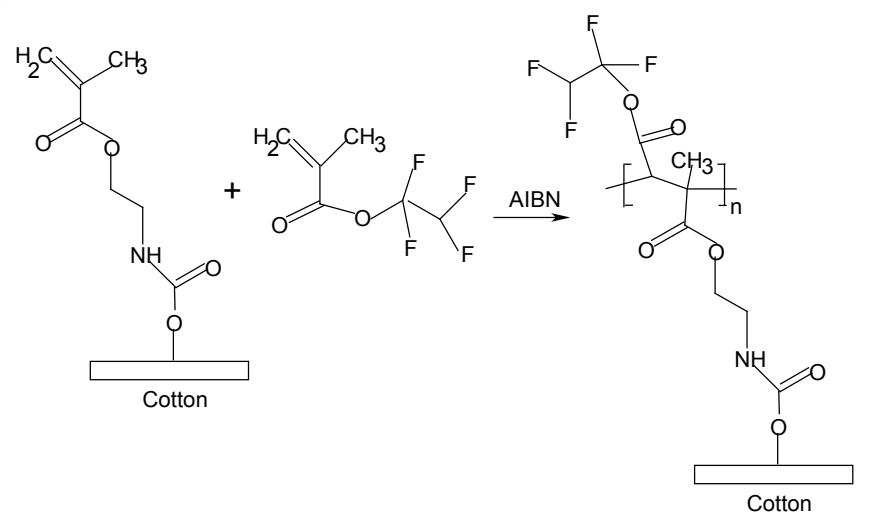

Scheme 2: Polymerization of fluorinated acrylic monomers on the surface of cotton.

samples were washed with acetone and with ethanol subsequently. The cotton samples were then dried in an oven at $70^{\circ} \mathrm{C}$.

\section{Characterization}

Attenuated Total Reflectance Fourier Transform Infrared (ATRFTIR): In order to determine the change in the functional groups after the chemical treatment of cotton fabric, Attenuated Total Reflectance Fourier Transform Infrared (ATR-FTIR) (Perkin Elmer Frontier) was used.

Contact angle: Contact angle (CA) measurements were carried out using Contact Angle Dataphysics OCA 20 (Dataphysics, Germany) instrument and $6 \mathrm{uL}$ deionized water droplets at room temperature. Five measurements at different positions on the cotton fabric were used for the determination of the average contact angle values. All the values reported were static contact angles, and measurements were taken 30 seconds after the water droplets were applied onto the cotton fabric.

Scanning Electron Microscopy (SEM)/Energy Dispersive X-ray Spectroscopy (EDX): Scanning Electron Microscopy (FESEM, JSM$6340 \mathrm{~F}$ ) was used to observe the morphology of cotton samples before and after treatment while the energy-dispersive X-ray spectroscopy EDX was carried out by JEOL-6360 under the scanning voltage of 20 $\mathrm{kV}$. The amount of fluorine contented was calculated through area mapping.

Mechanical analysis: The tensile test had been performed at room temperature with Mechanical Tester MTS C43. Each sample was prepared with a size of $10 \mathrm{~mm}$ by $40 \mathrm{~mm}$, with a thickness of around $0.25 \mathrm{~mm}$. A load cell of $100 \mathrm{~N}$ was used. Five measurements for each type of fabric were performed. 


\section{Results}

\section{Characterization of obtained cotton samples}

Successful deposition of hydrophobic coatings on the cotton sample was evaluated by comparing the FTIR spectra of coated cotton samples with the untreated sample. FTIR spectra of untreated and treated cotton fabrics are presented in Figure 1. The peak near 1730 $\mathrm{cm}^{-1}$ was attributed to the stretching vibration of the carbonyl group of acrylates monomers attached to the fabric. Because of a small amount of fluorinated polymer on the cotton surface and overlapping of peaks typical for $-\mathrm{CF}_{3}$ group, there are no further clearly distinguishable differences between treated and untreated cotton. All presented SEM photos were taken using cotton samples obtained using the ratio of $1: 1$ of- $\mathrm{OH}$ groups to fluorinated monomer because this ratio has shown higher values of a contact angle. The SEM image of the untreated cotton sample (Figure 2) had shown a smooth surface with characteristic parallel ridges. The primary wall structure was also obvious from the SEM image. The SEM image of the treated cotton samples did not exhibit the fine fibril structure that was present in the starting material. Thin laminates and patches with irregular edges were observed. These may have resulted from the rearrangement of surface cellulose due to the formation of a thin fluorinated layer in the primary wall. To characterize the chemical change of cotton after the treatment, quantitative EDX analysis of elements were made using the same samples chosen for SEM. The fluorine content found on the surface of the differently treated cotton is presented in Table 1 . As seen in Table 1, the amount of fluorine on the surface of cotton had increased from $0.46 \%$ for sample coated with TFPA up to $0.90 \%$ for the sample coated with DFHA. Theoretically, if all $-\mathrm{OH}$ groups were modified and covered by fluorinated monomers, the amount of fluorine in the treated cotton should be $6.2 \%$ wt. for TFPA and up to $12.9 \%$ wt. for DFHA. Therefore, the degree of fluorination of acrylated -OH group can be approximately estimated to be $7 \%$.

\section{Water repellent property}

The average contact angles of the differently treated cotton fabrics and different ratios of the number of double bonds on the cotton surface to the amount of fluorinated monomer are presented in Table 2. It should be noted that the untreated contact angle of cotton fabrics could not be measured because the water droplet was quickly absorbed into the fabric within 4 seconds. This is due to the high hydrophilic properties of cotton. The intermediate product, where acrylic groups are attached on the surface of cotton, has a contact angle

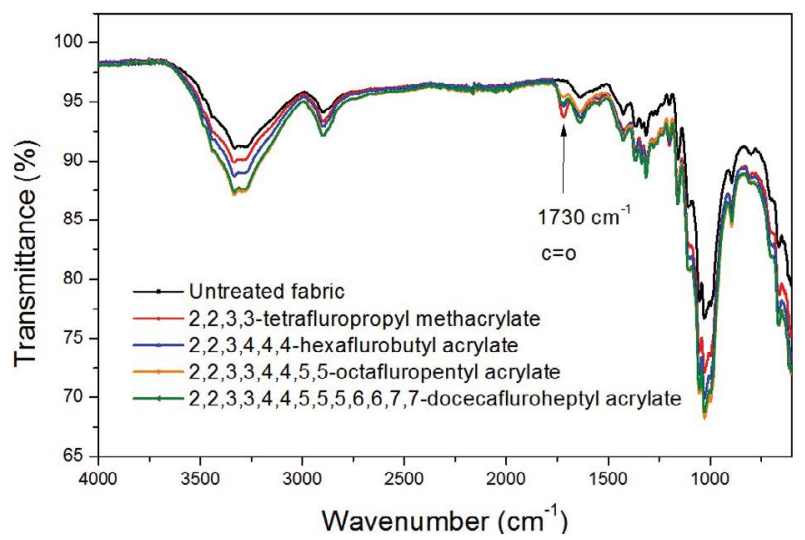

Figure 1: FTIR spectra of initial cotton fabrics treated with fluorine-containing monomers.
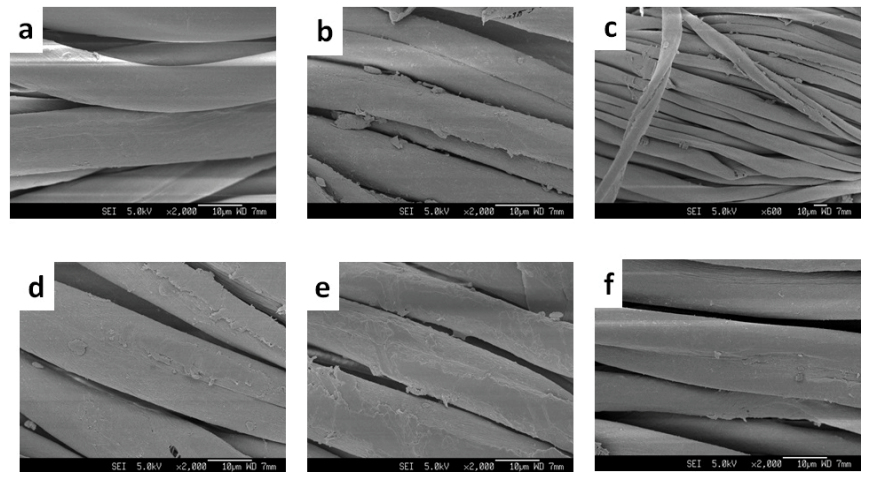

Figure 2: SEM pictures of a) initial cotton fabric and fabrics treated with b) 2,2,3,3-tetrafluoropropyl methacrylate c) high resolution of b), d) 2,2,3,4,4,4-hexafluorobutyl acrylate e) 2,2,3,3,4,4,5,5-octafluoropentyl acrylate f) $2,2,3,3,5,5,5,6,6,7,7$-docecafluoroheptyl acrylate.

\begin{tabular}{|c|c|}
\hline Type of fluorinated monomer & Amount of fluorine \% mass \\
\hline TFPA & 0.46 \\
\hline HFBA & 0.78 \\
\hline OFPA & 0.79 \\
\hline DFHA & 0.90 \\
\hline
\end{tabular}

Table 1: Amount of fluorine in the treated cotton samples.

\begin{tabular}{|c|c|c|c|}
\hline \multirow{2}{*}{$\begin{array}{c}\text { Type of fluorinated } \\
\text { monomer }\end{array}$} & \multicolumn{3}{|c|}{$\begin{array}{c}\text { Average contact angle }\left(^{\circ}\right) \text { Ratio of Double bond on } \\
\text { surface: Fluorinated monomer }\end{array}$} \\
\cline { 2 - 4 } & $\mathbf{1 : 1}$ & $\mathbf{1 : 5}$ & $\mathbf{1 : 1 0}$ \\
\hline TFPA & 107.7 & 103.6 & 89.5 \\
\hline HFBA & 113.2 & 105.5 & 101.9 \\
\hline OFPA & 123.6 & 122.6 & 107.8 \\
\hline DFHA & 128.2 & 122.8 & 115.4 \\
\hline
\end{tabular}

Table 2: Contact angle values for differently treated cotton fabrics.

of $98.3^{\circ}$, which confirmed that there had been a modification of the $-\mathrm{OH}$ groups. However, it was still lower than that of fluorine-treated cotton fabric, due to the absence of hydrophobic tail group (C-F). It can be seen that the cotton treated with longer chains of fluorinated monomers were more hydrophobic as compared to that with shorter chains. This might be due to nonpolar carbon-fluorine (C-F) chains decreasing the overall polarity of the molecule. In addition, longer carbon chains increase the hydrophobicity of the fabric surface, resulting in greater contact angle. As a general trend, the surface energy of cotton fabric decreases as the carbon chain lengths and amount of fluorine atoms increase. In addition, the introduction of long fluorine chains creates rougher surfaces on the cotton fibers, and this plays a positive role in hydrophobicity by decreasing the surface free energy as well. The increase in the molar ratio of the fluorinated monomer has an interesting influence on hydrophobicity. The higher amount of fluorinated monomers we add, the lower hydrophobicity of fabric we obtain. A large molecular chain on the cotton surface could create active sites between the fluorinated monomer chain and the substrate. In particular, a 1:10 molar ratio could create a multiple-linking system, as was mentioned by Colleoni [19], in which the influence of the fluorine chains steric hindrance is balanced by an increase in the number of links between the coating and the cotton fabric sample substrate. Long macromolecule with fluorine atoms on the surface of cotton is also more inclined to make some compact structures such as crystals, coils or globules where fluorine atoms could be hidden and become unavailable to impact hydrophobicity. 
Citation: Liu X, Yang G, Lipik VT (2017) Effects of Fluorine Atoms Amount and Fluorinated Acrylic Chain Length Chemically Attached to Hydroxyl Groups on the Hydrophobic Properties of Cotton Fabrics. Mod Chem Appl 5: 204. doi: 10.4172/2329-6798.1000204

Page 4 of 4

\section{Mechanical properties of modified fluorinated cotton}

It had been observed that there were slight differences between the mechanical properties of untreated and treated cotton. Young moduli of all samples were fluctuating within the same range. Untreated cotton had the lowest modulus $\left(201.0 \mathrm{~N} / \mathrm{mm}^{2}\right)$ while the sample with TFPA treatment had shown the highest $(328.4 \mathrm{~N} / \mathrm{mm})$. The treated samples showed decreasing modulus with increasing number of fluorine up to $272.0 \mathrm{~N} / \mathrm{mm}^{2}$ for DFHA treated sample. Measurements of the mechanical properties of the untreated and treated cotton fabrics are given in Figure 3. From the graphs, it could be seen that samples of fabric became stiffer after treatment, with the maximum stress value being $60 \%$ higher for fabrics treated with TFPA as compared to the untreated fabric. It had also been observed that the treated cotton fabrics had a loss in strain and elongation at break values almost twice as compared to untreated fabric. It could be related to the adhesion between cotton fibers. In addition, the formation of polymeric bridges between cotton fibers due to polymerization could also be possible. This phenomenon had also been mentioned by Maity [15]. An interesting observation was the value of stress connected with the amount of fluorine atoms in monomers used for treatment. Monomers with higher fluorine content used for treatment leads to lower stiffness and stress value of the fabric. It could likely be related to the crystallinity of polymer formed on the cotton surface. Lower fluorine content and shorter length of monomer tends to result in higher crystallinity at the same degree of polymerization.

\section{Conclusion}

A thin coating with fluorinated polymer chemically attached to the cotton fabric was shown. The amount of fluorine in the final fabric is lower than $1 \%$ wt, which is lower when compared to other similar treatments, and yet the fabric is showing good water-repellent property. The cotton that was treated with $2,2,3,3,4,4,5,5,5,6,6,7,7$-docecafluoroheptyl acrylate obtained the highest contact angle of $128^{\circ}$. Although modification of cotton can allow for better hydrophobicity, mechanical properties need not be sacrificed. It had been found that by making the length of the attached polymer longer, not only will the mechanical properties not be affected significantly but at the same time it gives the best result in terms of hydrophilicity. It can also be said that there will be a smaller environmental impact as less fluorine will go into the environment during the utilization steps of fluorinated cotton fabric

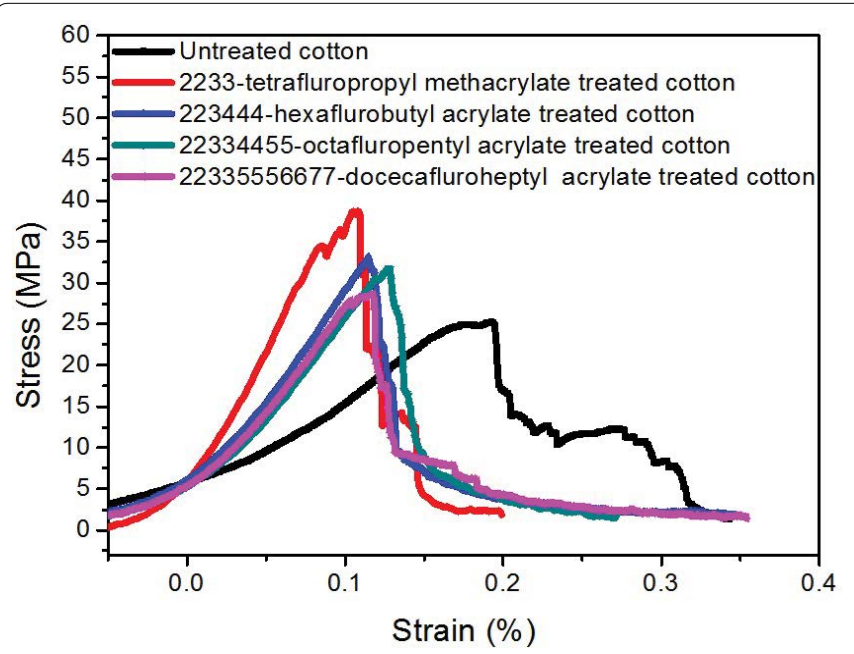

Figure 3: Stress-strain plot of untreated cotton fabric and fabric after treatment with different fluorine-containing monomers. as well as the usage. The developed method opens a wide area allowing for coating with layers, using a combination of acrylic monomers for coating, making different lengths of hydrophobic polymers on the cotton, and creating other properties, for example antimicrobial, of fabric.

\section{References}

1. Xue CH, Jia ST, Zhang J, Tian LQ, Chen HZ, et al. (2008) Preparation of super hydrophobic surface on cotton textile. Sci Tech of Adv Mat 9: 1-7.

2. Shosha MH, Hilw ZH, Aly AA, Amr A, Al I, et al. (2008) Paraffin wax emulsion as water repellent for cotton/polyester blended fabric. J Ind Text 37: 315-325.

3. Przybylak M, Maciejewski H, Dutkiewicz A, Dabec I, Novicki M, et al. (2016) Fabrication of superhydrophobic cotton fabric by a simple chemical modification. Cellulose 23: 2185-2197.

4. Li Y, Zheng X, Xia Z, Lu M (2016) Synthesis of fluorinated block copolyme and superhydrophobic cotton fabric preparation. Prog Org Coat 97: 122-132.

5. Holmquist $\mathrm{H}$, Schellenberger SI, Vander V, Peters GM, Leonards PEG, et al. (2016) Properties performance and associated hazards of state of the art durable water repellent (DWR) chemistry for textile finishing. Environment International 91: 251-264.

6. McKeen LW (2006) Fluorinated coating and finishes handbook. Wiliam Andrew Publication.

7. Mahltig B, Bottcher H (2002) Modified Silica Sol Coatings for Water-Repellent Textiles. J Solgel Sci Technol 27: 43-52.

8. Basu BJ, Bharathidasan T, Anandan C (2013) Superhydrophobic oleophobic PDMS-silica nanocomposite coating. Ice Science 1: 40-51.

9. Chen W, Fadeev AY, Hsieh MC, Oner D (1999) Ultra hydrophobic and Ultra Lyophobic Surfaces: Some Comments and Examples. Langmuir 15: 33953399.

10. Yao W, Li Y, Huang $X$ (2014) Fluorinated polymethacrylate: Synthesis and properties. Polymer 55: 6197-6211.

11. Schondelmaier D, Cramm S, Klingeler R, Morenzin J, Zilkens C, et al. (2002) Orientation and Self-Assembly of Hydrophobic Fluoroalkylsilanes. Langmuir 18: $6242-6245$.

12. Yang Y, Shen J, Zhang L, Li X (2015) Preparation of a novel water and oilrepellent fabric finishing agent containing a short perfluoroalkyl chain and its application in textile. Mater Res Innov 19: 401-404

13. Shi Z, Wyman I, Liu G, Hu H, Zou H, et al. (2013) Preparation of water-repellent cotton fabric from fluorinate di block copolymers and evaluation of their durability. Polymer 54: 6406-6414.

14. Cai R, Deng B, Jiang H, Yu Y, Yu M, et al. (2012) Radiation induced graft polymerization of a fluorinated acrylate onto fabric. Radiat Phys Chem 81 1354-1356.

15. Maity J, Kothary P, Rear EA, Jacob C (2010) Preparation and comparison of hydrophobic cotton fabric obtained by direct fluorination and micellar polymerization of fluoro monomers. Ind Eng Chem Res 49: 6075-6079.

16. Yu Y, Wang Q, Yuan J, Fan X, Wang P, et al. (2016) Hydrophobic modification of cotton fabric with octadecylamine via laccase/TEMPO mediated grafting Carbo hyd. Polym 13: 549-555.

17. Grajeck EJ, Petersen WH (1962) Oil and water repellent fluorochemical finishes for cotton. Textile Research Journal 32: 320-331.

18. Kumar UN, Kratz K, Wagermaier W, Behl M, Lendlein A, et al. (2010) Noncontact actuation of triple-shape effect in multiphase polymer network nanocomposites in alternating magnetic field. J Mater Chem 20: 3404-3415.

19. Colleoni C, Guido E, Migani V, Rosace G (2015) Hydrophobic behaviour of non-fluorinated sol-gel based cotton and polyester fabric coatings. J Ind Text 44: 815-834 